\title{
Enabling Silicon-on-silicon photonics with pedestalled Mie resonators.
}

\author{
M. Garín, ${ }^{*}$ M. Solà, A. Julian and P. Ortega \\ Grup de recerca en Micro i Nanotecnologies, Departament d'Enginyeria Electrònica, \\ Universitat Politècnica de Catalunya, c/ Jordi Girona Pascual 1-3, Barcelona 08034, Spain \\ * Corresponding author: moises.garin@upc.edu
}

\begin{abstract}
High-refractive-index Mie resonators are regarded as promising building blocks for low-loss all-dielectric nanophotonic applications. To avoid the otherwise excessive damping and loss of symmetry such devices typically need to be implemented over a low-index substrate, which hampers their integration in many practical applications. In this paper we propose a new photonic structure consisting of silicon-on-silicon spheroidal-like resonators, each one supported by a slim silicon pedestal that makes the micro-cavities stand optically separated from the substrate while providing both mechanical stability and electrical contact with the substrate. These structures are produced in high-quality monocrystalline Si and their size and arrangement can be precisely controlled through standard lithography. We demonstrate that such structures present an optical performance similar to the one achieved with low-index substrates, opening new avenues for developing novel hybrid photonic/electronic devices.
\end{abstract}

\section{Introduction}

All-dielectric nanophotonics is rapidly emerging as a promising alternative to plasmonics due to its exciting properties and the prospect of operating with reduced optical losses and reduced heat dissipation. ${ }^{1}$ These structures rely on high-refractive-index (HI) optical micro- and nano-cavities featuring strong electric and magnetic multipolar resonances that overlap in certain frequency 
ranges. ${ }^{2,3}$ Such nanoresonators are used as building blocks (or photonic atoms) in a plethora of applications, ${ }^{4}$ often through particular surface ensembles (so-called metasurfaces) that allow them to couple in the near-field. Some application examples for all-dielectric metasurfaces include perfect reflectors, ${ }^{5}$ magnetic mirrors, ${ }^{6-8}$ metalenses, ${ }^{9}$ beam manipulation, ${ }^{10}$ efficient Huygens metasurfaces ${ }^{11}$ and controlling the Brewster effect, ${ }^{12}$ just to name a few.

Classical semiconductors, such as Si, are considered amongst the best suited materials for alldielectric photonics due to their high refractive index $\left(n_{\mathrm{Si}} \geq 3.42\right)$, low (or relatively low) losses in the NIR $(\lambda>750 \mathrm{~nm})$, the broad spectrum of available nano-fabrication techniques, and the anticipation of MOS compatibility and integration in hybrid photonic/electronic devices. Among all possible geometries, spherically shaped particles are particularly appealing due to their high symmetry giving rise to pure Mie resonances including both strong magnetic and electric dipole resonances as well as higher $Q$-factor higher-order resonances and whispering gallery modes. During the past decade, several routes have been devised for producing such silicon spherical particles in the micrometre and submicrometre range such as high-temperature trisilane thermolysis in supercritical solvents, ${ }^{13,14}$ gas-phase synthesis by Chemical Vapor Deposition (CVD), ${ }^{15-17}$ laser ablation, ${ }^{3,18,19}$ or in-fibre capillary instabilities. ${ }^{20}$ These methods excel in different respects such as the excellent spherical quality and material purity achieved by CVD, the good size control and dispersion with trisilane thermolysis, the ability to set the particle position by laser ablation or the good size scalability in the fibre instability method. A myriad of applications have been already sought for these high-quality resonators, like in communications, ${ }^{21-26}$ as sunlight harvesters, ${ }^{27,28}$ for UV/VIS/IR blocking, ${ }^{29}$ or to achieve high surface enhancement of both fluorescence and Raman scattering, ${ }^{30-33}$ among others.

In spite of the advantages of spherical cavities, the production and processing of monodisperse high-index micro-/nano-spheres still poses a serious challenge that has largely prevented their use in many applications including metasurfaces. Furthermore, HI spherical nanoparticles are largely perceived as an idealized, and somewhat exotic, approach that is not ready for real world applications. Instead, practical realizations substitute spheres by disks, or similar nanostructures, that still exhibit the fundamental Mie resonances (magnetic and electric dipoles) and that can be easily produced through standard lithography and microfabrication techniques. However these geometries are highly sensitive to the substrate material and, therefore, need to be produced on a low-refractive index substrate (e.g. $\mathrm{SiO}_{2}$ or $\mathrm{BaF}_{2}$ ) in order to avoid an excessive optical damping, loss of symmetry, and alteration of the resonances. ${ }^{34}$ 
In this paper we propose a new structure consisting of monocrystalline silicon-on-silicon spheroidal-like resonators, each one supported by a slim pedestal that makes the micro-cavities stand optically separated from the substrate as well as providing mechanical stability and electrical contact with the substrate. This optical separation allows higher order Mie resonances to survive in addition to the fundamental ones, producing astonishing optical properties as expected for metasurfaces even though they are built onto a substrate of the same high refractive index. These structures are obtained by combining lithography and high-temperature solid-phase reorganization techniques. Consequently, the whole structure, spheroid, pedestal, and substrate are made of the same, high-quality monocrytalline silicon while the location and size of the spheroids can be fully defined at the lithography step. Moreover, due to the scalability of the surface reorganization process, the proposed structure can potentially be scaled down to the nanometer range. These characteristics offer new possibilities for developing high-quality, very low loss, on-chip silicon photonics.

\section{Results}

Figure 1 shows different examples of the fabricated pedestalled structures than can be achieved with the proposed method. Pedestalled monocrystalline-silicon spheroid-like particles have been produced through high-temperature transformation of silicon pillars (See fig 1a,b), which were produced on a crystalline wafer through standard lithography and dry etching techniques. By annealing the wafer at temperatures above $1100{ }^{\circ} \mathrm{C}$ in deoxidizing ambient (typically $\mathrm{H}_{2}$ or $\mathrm{Ar}$ ) pillars evolve in solid phase by surface diffusion, tending to minimize the surface curvature, and leading to Rayleigh instabilities for high enough pillars. By precisely stopping the annealing before the pillar actually pinches off, one can produce pedestalled spheroid-like structures with a narrowneck union. This process is shown schematically in Fig. 1a, where the transformation has been numerically calculated by finite-difference time-domain (FDTD) method assuming an isotropic surface diffusion process. Further details about the theory and simulation of the pillar transformation are given in the methods section. Figure $1 \mathbf{b}$ shows a scanning electron microscope (SEM) image of real pedestalled silicon spheroids obtained after annealing for 90 min at $1100{ }^{\circ} \mathrm{C}$ in $\mathrm{Ar}+\mathrm{H}_{2}(5 \%)$ ambient a square array (4 $\mu \mathrm{m}$ pitch) of pillars with a diameter of $2 \mu \mathrm{m}$ and an aspect ratio of 3. Since the whole process occurs in solid phase by surface diffusion, the entire structure maintains the single crystal nature, as can be evidenced by main crystalline faces developing at the surface of the spheroids. A further discussion about the monocrystallinity of the structure can be found in the supplementary material, including experimental identification of the emerging 
crystalline facets and microstructure characterization of individual particles through Raman spectroscopy. ${ }^{35}$
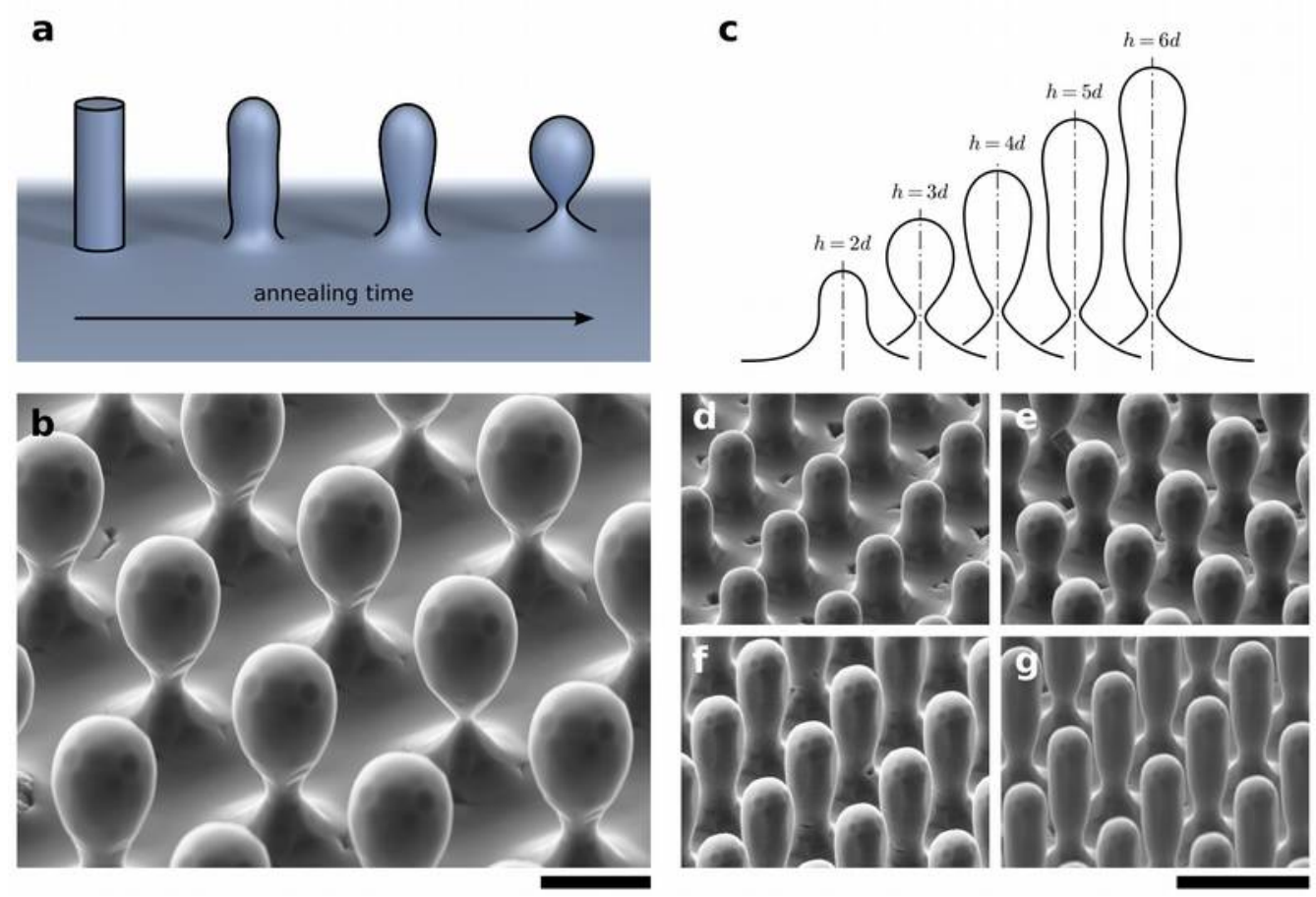

Fig. 1. (a) Schematic of the transformation of a pillar during a high-temperature annealing. The shapes have been obtained through numerical calculations assuming an isotropic surface diffusion process. (b) SEM image of a square array of spheroid-like structures. The scale bar is $2 \mu \mathrm{m}$. (c) Cross-sectional shapes (numerical calculations) obtained after annealing silicon cylindrical pillars with different initial aspect ratios. (b-e) SEM images of different structures obtained after annealing a square array of silicon pillars of increasing height. The scale bar, common to the four images, represents $5 \mu \mathrm{m}$.

Transformation by surface diffusion is a scale independent process and, therefore, the resulting shape solely depends on the initial aspect ratio $(h / d)$ of the pillars and the total annealing time. ${ }^{36,37}$ Smaller structures can be obtained just by scaling down the size of the initial pillars. In the supplementary material we show preliminary results for $1 \mu \mathrm{m}$ pedestalled spheroids along further comments about scaling the structures into the sub-micrometre range. A minimum aspect ratio is needed for the Rayleigh instability to appear. ${ }^{38}$ Numerical calculations (see Fig. 1c) show that, for isolated cylindrical pillars under isotropic surface diffusion, instabilities appear for an aspect ratio $h / d=3$, roughly. Pillars with aspect ratios shorter than the threshold will just end up being reabsorbed by the substrate during the annealing. A very interesting property of the process is that the instability always develops first on the base of the pillar regardless of its height, as calculations in Fig. 1c clearly show. Furthermore, the time to pinch-off is nearly independent of the pillar height provided that the pillar diameter is the same. This allows to fabricate structures with different 
shapes ranging from pedestalled spheroids, by using aspect ratios close to the threshold, to structures resembling rod cells of different heights for larger aspect ratios. As an example, Fig. 1d-g shows SEM images of different arrays of pillars with increasing pillar height and after the exact same annealing time in $\mathrm{Ar}-\mathrm{H}_{2}$ (5\%) ambient. Even further, it is conceivable to produce complex mixed structures (e.g. structures simultaneously including spheroids and rods) since the neck is formed after a similar annealing time.

The presented fabrication method relies on standard lithography and dry etching techniques in order to define the location and size of the structures. This makes it particularly well suited to produce particle ensembles such as, for instance, metasurfaces. In fact, square arrays of spheres such as the arrangement shown in Fig. 1 but on a low index substrate exhibit notable optical features expected in high-dielectric metasurfaces such as, for instance, high transmittance, ${ }^{40,41}$ perfect reflectance ${ }^{5,39}$ polarization switchable high reflectance/transmittance, ${ }^{42}$ magnetic reflectance, ${ }^{8}$ and generalized Brewster effect. ${ }^{12}$ In order to reveal the potential of pedestalled structures, in Figure 2 we show a detailed experimental characterization of the optical reflectance of a metasurface composed by a square array of pedestalled spheroids obtained by annealing pillars with an initial aspect ratio of $h / d=3$. Figure 2a shows a SEM image of the actual sample measured. The array has a pitch of $4 \mu \mathrm{m}$ and the initial pillars were fabricated targeting a diameter $d=2 \mu \mathrm{m}$ and a height $h=6 \mu \mathrm{m}$. These dimensions lead to magnetic and electric dipoles in the mid-IR at wavelengths around $8 \mu \mathrm{m}$, which can be characterized by Fourier Transform Infrared (FT-IR) spectroscopy. The specular reflectance spectra at quasi-normal incidence $\left(13^{\circ}\right)$ is shown in figure $2 \mathrm{~b}$, where a wide total reflection band due to the combined effect of the electric and magnetic dipoles $^{5}$ can be observed. For comparison purposes the experimental reflectance of the structure before annealing (array of silicon pillars) is also shown in dots, missing any interesting features. In figure 2c,d we also show the experimentally measured specular reflectance as a function of the incidence angle for both light polarizations. The diffraction limit is shown in dashed lines as a guide, the subdiffractive regime being to the left of that line. Here we can see that the high reflection band feature is mostly independent of the angle of incidence, as one would expect from a resonator with high spherical symmetry. We will further discuss this later on the light of scattering calculations for a single pedestalled spheroid. The reflectance maps in figure 2c,d also seem to exhibit the recently reported generalized Brewster effect, ${ }^{12}$ especially at the edges of the high reflection band. In fact, the experimental reflectance maps shown here closely resemble the theoretical calculations presented in that work $^{12}$ for an array of spheres in air, even though our produced spheroid-like resonators were a bit smaller than expected what pushed the features 
slightly towards the diffractive region. The generalized Brewster effect can be better visualized at the panels e-g in Fig. 2, which show the reflectance vs angle for both polarizations and at selected wavelengths. Here we can see that the null reflectance can be achieved for different wavelengths and polarizations within the subdiffractive regime. Notice also that, in these curves, reflection cannot reach zero as we must consider the reflection at the polished back surface of the silicon samples.

a
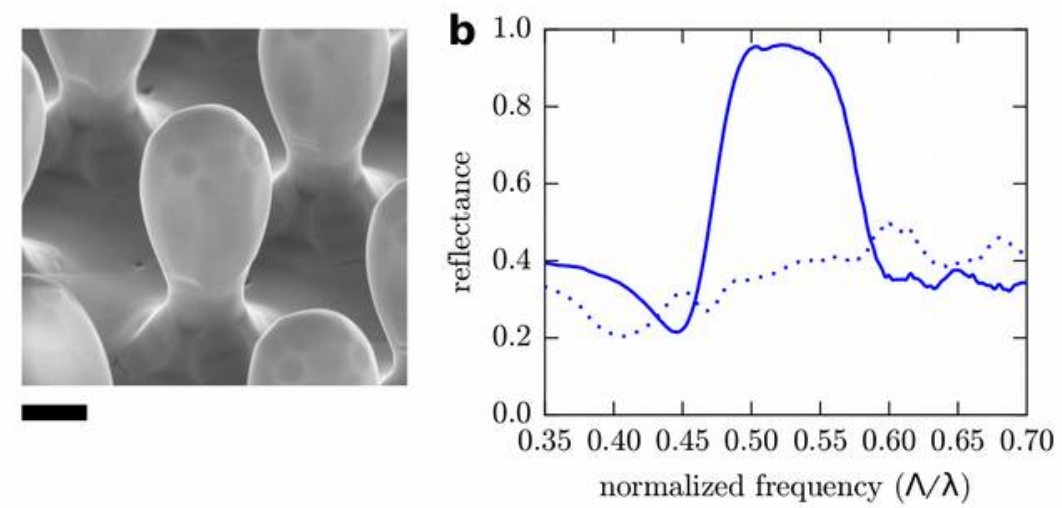

C wavelength $(\mu \mathrm{m})$
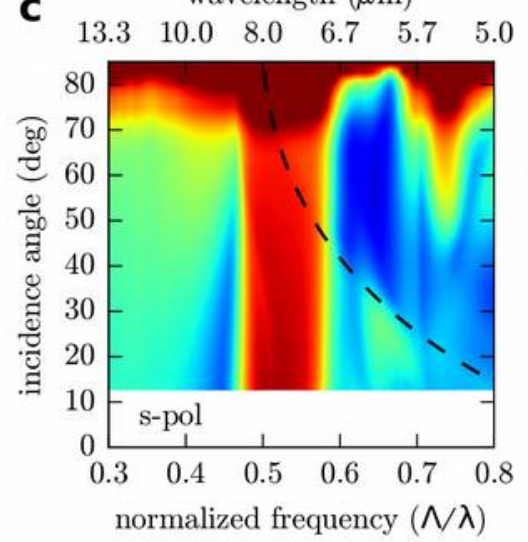
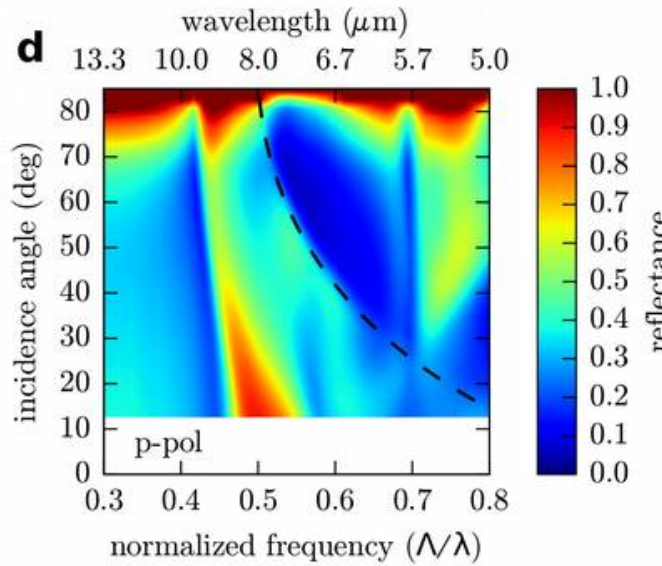
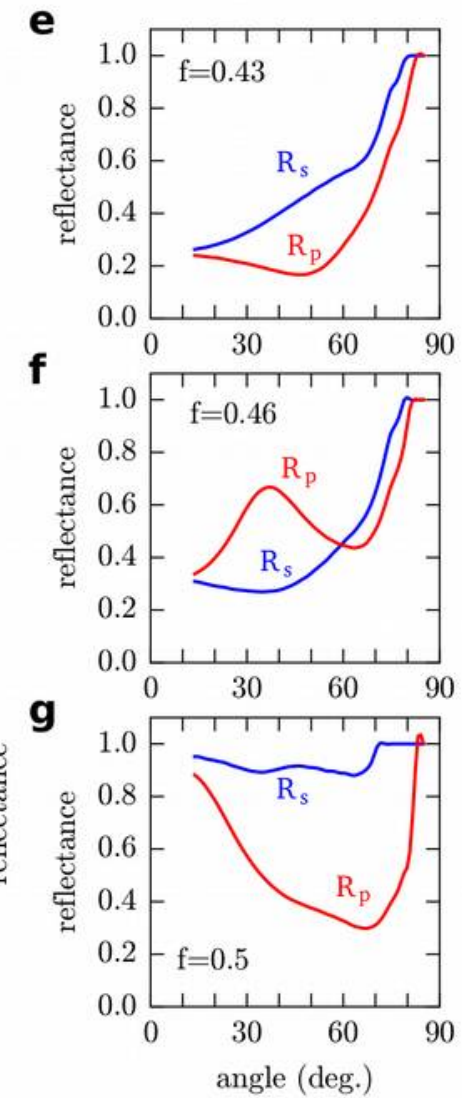

Fig. 2. (a) SEM image, detail of a single pedestalled spheroid, of the measured sample. It consists of a square array, $4 \mu \mathrm{m}$ pitch, of pedestalled spheroids obtained after annealing pillars with, roughly, $d=2 \mu \mathrm{m}$ and $h=6 \mu \mathrm{m}$. The scale bar is $1 \mu \mathrm{m}$. (b) Specular reflectance (solid line) of the sample at quasi-normal incidence $\left(13^{\circ}\right)$. As a reference, the reflectance of the pillar array before annealing is shown in dots. (c,d) Specular reflectance vs incidence angle color maps for both $\mathrm{p}$ and $\mathrm{s}$ polarizations. The dashed line represents the limit of the diffractive region. $(\mathbf{e}-\mathbf{g})$ Reflectance vs. angle for both $\mathrm{p}$ and $\mathrm{s}$ polarization at selected normalized frequencies. 


\section{Discussion}

In the previous section we have shown that pedestalled-based metasurfaces show an outstanding performance, similar to what would be obtained with silicon spheres without substrate. These results were obtained with a sample that did not exhibit a particularly narrow neck. In order to better understand how important is the size and shape of the neck on the performance of the pedestalled resonators, we have calculated by rigorous coupled-wave analysis (RCWA), ${ }^{48}$ see methods section for further details, the total reflectance at normal incidence of a 2D array of pedestalled spheroids at the different stages of transformation (numerical calculations) shown in figure 3a: from the initial pillar at $t_{0}$, up to the close to pinch-off spheroid at $t_{5}$. Notice that the snapshots shown in fig. 3a do not follow constant time increments but have been selected according to the progression of the overall shape. Reflectance results are shown in figure $3 c$ for a square array distribution of spheroids as shown in figure 3b. First of all, the differences in reflectance between the initial pillar structure at $t_{0}$ and the final spheroidal-like structure at $t_{5}$ are dramatic. The array of cylindrical pillars presents a reflection spectra that is rather smooth oscillating between 0 and $30 \%$ (roughly). On the contrary, the array of pedestalled spheroids exhibits a wide perfect reflection band around a frequency of 0.5 (nu), due to the magnetic and electric dipole resonances, as well as sharp reflection peaks and features due to higher order resonances. Simulations also show that an abrupt change in the optical behaviour of the structure occurs at the spheroidal-like shape obtained between $t_{2}$ and $t_{3}$. While at $t_{2}$ just the large reflection band starts to raise, at $t_{3}$ all relevant features are present at the reflectance spectrum. Beyond $t_{3}$ the features tend to strengthen and some slightly shift. At $t_{3}$ the pillar has already attained its distinctive tear-like shape, with a maximum diameter around $1.3 \times d$, while at the neck the diameter has shrunk down to $0.5 \times d$. It is for us quite remarkable that the spheroids show a good substrate decoupling with just a 50\% reduction of the diameter at the neck, with respect to the initial pillar, which proves the robustness of these structures with respect to process variations. 

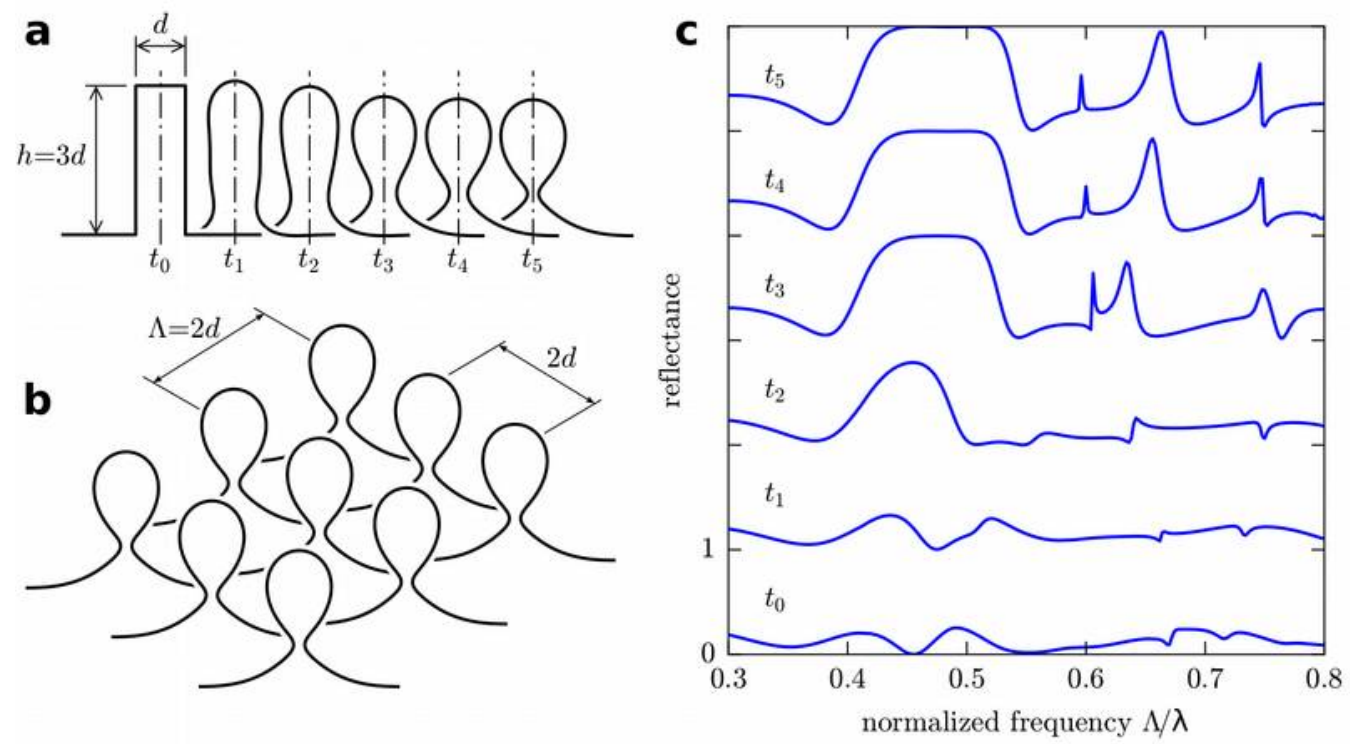

Fig. 3. (a) Time evolution (numerical calculations) of a silicon cylinder during annealing. The time snapshots do not correspond to equal time increments. The time $t_{0}$ corresponds to the initial cylinder before annealing. (b) Schematic of the studied metasurface; a square array, $\Lambda=2 d$, of pedestalled spheroids. (c) Calculated reflectance at normal incidence of a metasurface considering the different pedestalled shapes in panel (a).
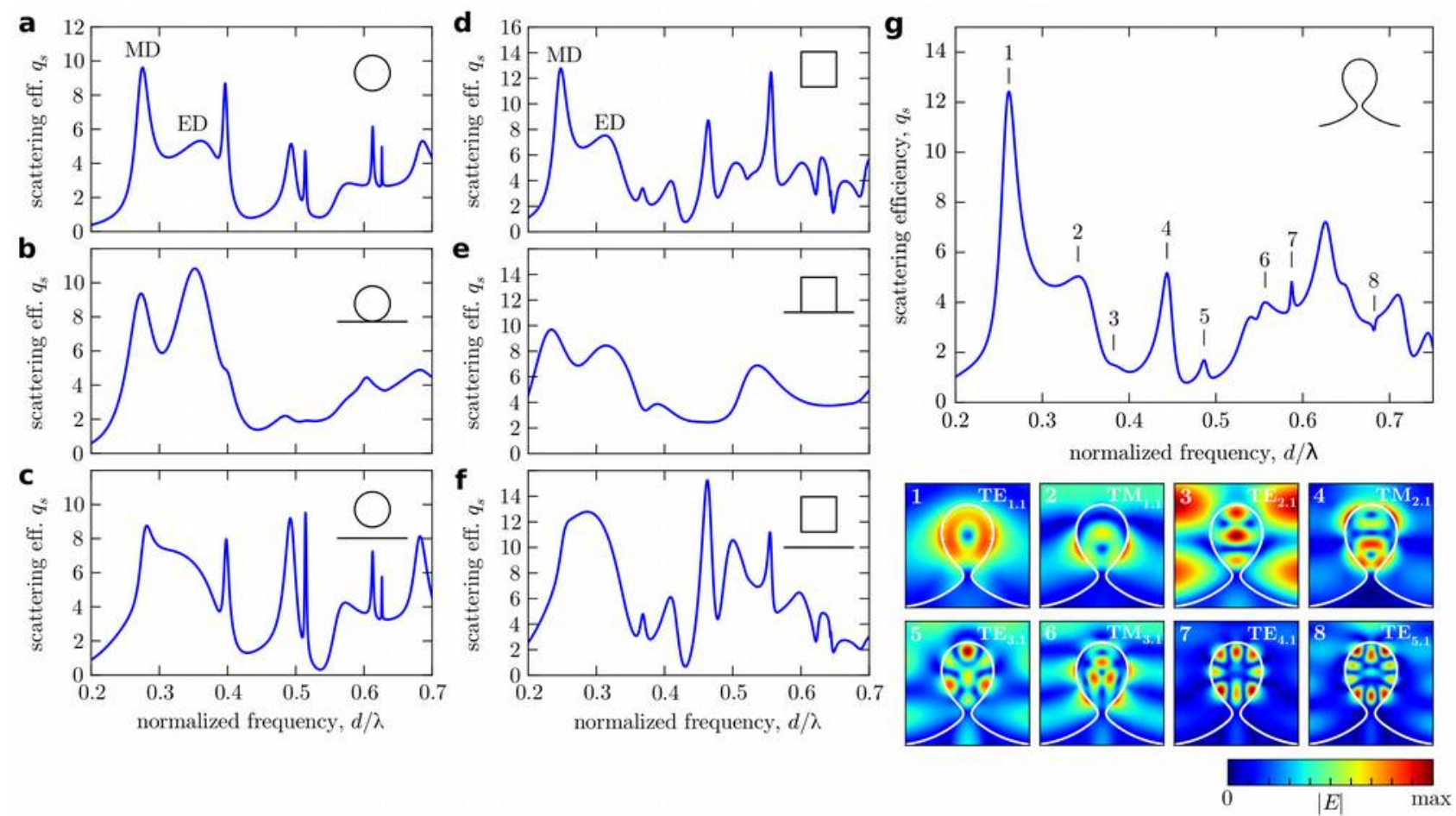

Fig. 4. Scattering efficiency at normal incidence of a silicon sphere (a) in air, (b) over a silicon substrate and (c) at a distance from a silicon substrate. (d to f) Scattering efficiency at normal incidence of a silicon cylinder with height equal to the diameter in air, over a silicon substrate and at a distance from a silicon substrate, respectively. (g) Scattering efficiency of a single pedestalled silicon spheroid. The colour panels show the electric field distribution (|E|) of selected resonant modes. Colour panels are independently normalized to the maximum $|E|$ value. All calculations were obtained by FDTD (see methods section). 
In order to better understand why pedestalled spheroids decouple so efficiently from the substrate, it is worth revisiting by way of example the performance of high-index resonators in the worst-case scenario, that is over a substrate of the same high-index material and under normal incidence. Figure 4 shows the scattering cross section (FDTD calculations, see methods section for further details) at normal incidence for two common scattering geometries, namely an ideal silicon sphere and a more practical silicon nanodisk in different positions relative to a silicon substrate. In particular, the figure shows the scattering of an isolated scatterer in air, over a silicon substrate, and at a distance $r$ above the substrate, where $r$ is the radius of the sphere or cylinder. In the ideal case of a scatterer embedded in air (Fig. 4a,d), the spectra of both the sphere and the cylinder exhibit clear electric and magnetic multipolar resonances, with the lowest order modes corresponding to magnetic and electric dipoles (respectively $\mathrm{MD}$ and $\mathrm{ED}$ in Fig. 4). In contrast, placing the resonators over a high-index substrate (Fig. 4b,e) in essence erases all resonances but the fundamental ones (magnetic and electric dipoles) from the scattering spectrum. At normal incidence the plane of resonance is perpendicular to the substrate, which acts as a strong loss mechanism killing all higher-order high $Q$-factor resonances. ${ }^{43}$ This loss mechanism is stronger for increasing contact area between the particle and substrate and, as a consequence, it becomes even more dramatic for the cylinder than for the sphere, affecting even the fundamental resonances. As a matter of fact, arrays of silicon cylinders etched over silicon have been already proposed as Miebased antireflective coatings, maximizing the scattering into the substrate, and producing a black surface to the naked eye. ${ }^{44}$ By allowing a gap half the size of the scatterer between the substrate and the scatterer (Fig. 4c,f) all high order features are again restored in the spectra. Actually, many higher order peaks start to resurface in the scattering spectra with just a separation of $10 \%$ the particle size $(0.2 \times r)$. In any case, multiple scattering effects by the sphere and plane still play an important role on the scattering spectrum, and a particular gap distance can enhance or weaken certain modes.

As we have shown, optical decoupling between the resonator and the substrate can be achieved by physically separating them, most sensibly by introducing a low-index spacing layer that may also work as antirreflective coating. ${ }^{45}$ Our strategy, however, proposes standing on a pedestal naturally appearing during the structure formation while keeping electrical and mechanical contact with the resonator. Figure $\mathbf{4} \mathbf{g}$ shows the calculated scattering efficiency of a single pedestalled spheroid at normal incidence, where it can be seen that the spectrum is much richer than that of the sphere and the pillar standing directly on silicon (see Fig. 4b,e), exhibiting clear higher order resonances in addition to the strong fundamental magnetic and electric dipoles. Surprisingly, and 
despite the ovate shape of the structure, many of these resonant features resemble typical Mie resonances of an ideal sphere in air. This can be better appreciated by looking at the field distributions shown at the bottom of Fig. $\mathbf{4} \mathbf{g}$ for selected resonances. These selected modes have been named according to the Mie resonance they approximate the most as $\mathrm{TE}_{\mathrm{m}, \mathrm{n}}$ and $\mathrm{TM}_{\mathrm{m}, \mathrm{n}}$ respectively for transverse electric (usually also named $b_{\mathrm{m}, \mathrm{n}}$ ) and transverse magnetic (also $a_{\mathrm{m}, \mathrm{n}}$ ) resonances, where $m$ is the mode number and $n$ is the order number. TE modes are associated to magnetic resonances, whereas TM modes are associated to electrical resonances. The pedestalled spheroids exhibit very clear magnetic multipolar resonances but for a very weak magnetic quadrupole $\left(\mathrm{TE}_{2,1}\right)$. The magnetic dipole $\left(\mathrm{TE}_{1,1}\right)$ is particularly strong and comparable to the magnetic resonance of spheres or disks in air. It is also worth mentioning the sharp features of both the magnetic octapole and decapole, although here the last manifests as a scattering dip. The electric resonances, on the other hand, seem more modified by the ovate shape of the structure and show an electric field distribution that barely resembles the familiar Mie resonances in a sphere. Nevertheless, the structure still presents a strong electric dipole and, especially, a very strong electric quadrupole. This good resonant behaviour of the pedestalled resonators explain the excellent results and the close performance pedestalled metasurfaces to their idealized spheres-inair counterparts.

\section{Conclusions}

We have introduced a new silicon photonic structure consisting of pedestalled silicon spheroids over a silicon substrate. These spheroid-like structures are obtained by transformation of silicon pillars through high temperature annealing, which gives them a characteristic pedestalled shape. In addition, since the transformation occurs by surface diffusion, the whole structure is made of highquality monocrystalline silicon and its size and location can be precisely controlled through standard lithography. By being raised over the surface through the pedestal, the spheroid-like resonators get decoupled from the substrate and can sustain resonances similar to silicon resonators over a low-index substrate although the spheroids maintain physical and electrical contact with the substrate. Square arrays of pedestalled spheroids are fabricated and characterized in this work, showing that metasurface effects such as total reflection, magnetic reflection and generalized Brewster effect are present in the structures. Experimental results show a performance similar to published results based on high-index cylinders over a low-index substrate. The presented structure opens new avenues for developing integrated hybrid photonic/electronic devices and for potentially lessening the gap for MOS integration. 


\section{Experimental section}

Fabrication of the arrays of pedestalled spheroids. Silicon pillars were created as follows. Base material are $<100>$ oriented, double side polished, $n$-type crystalline Si wafers. First, a 2D array of chromium dots was created over the bare silicon surface through standard lithography followed by $50 \mathrm{~nm}$ Cr deposition and a lift-off process. We typically used a photomask defining a square array of $2 \mu \mathrm{m}$ openings with $2 \mu \mathrm{m}$ spacing. Then, the wafer undergoes a vertical dry-etching step in an Oxford Plasmalab 100 ICP180 system using a mixture of $\mathrm{SF}_{6}$ and $\mathrm{C}_{4} \mathrm{~F}_{8}$ gases. The $\mathrm{Cr}$ dots act as mask during the etching, resulting in the formation of an array of Si pillars with vertical sidewalls. The Cr layer is subsequently etched away from the roof of the pillars by using a commercial $\mathrm{Cr}$ etching solution. Finally, the samples are annealed at ambient pressure in a horizontal quartz tube oven at $1100{ }^{\circ} \mathrm{C}$. A pure ambient of $\mathrm{Ar} 95 \%+\mathrm{H}_{2} 5 \%$, with very low $\mathrm{O}_{2}$ partial pressure, is maintained during the process by constantly flowing $10 \mathrm{slpm}$ of $\mathrm{Ar} / \mathrm{H}_{2}$ mixture. These ambient and temperature conditions are known to deoxidize the silicon surface and to promote the surface diffusion mechanism. The annealing time (typically in the range $1 \mathrm{~h}-2 \mathrm{~h}$ ) was optimized so that the transformation process is stopped before the pinch-off of the pillar occurs due to the Plateau-Rayleigh instability. Typical sample size ranged $1-4 \mathrm{~cm}^{2}$.

Calculations of the pillar transformation during annealing. Calculations of the pillar transformation during the high-temperature annealing were performed by considering isotropic surface diffusion as the main transformation mechanism, which is a good approximation at the process conditions. Surface diffusion is a process driven by curvature changes, where adatoms migrate from high curvature regions toward lower curvature ones reducing both quick curvature changes and the overall surface free energy. This transformation process can be described by the

Mullins' equation for surface diffusion: ${ }^{36} \frac{d n}{d t}=B \Delta_{s} h$, where $d n / d t$ is the rate of advance of a surface element in the normal direction, $B$ is a constant, $\Delta_{\mathrm{s}}$ is the Laplace-Beltrami operator, and $h=1 / 2\left(\kappa_{1}+\kappa_{2}\right)$ is the surface mean curvature, with $\kappa_{1}$ and $\kappa_{2}$ the principal curvatures. The Mullins' equation for surface diffusion was solved in time following a Finite-Difference Time-Domain (FDTD) approach using a dimensionless cylindrical symmetry formulation. ${ }^{37,38}$ Calculations were done using in-house developed FDTD software. The pillar profile was described with a series of control points which were then evolved through the FDTD process. In order to ensure the stability of the algorithm, points were dynamically added or removed depending on the distance with neighbouring points. ${ }^{46}$ Finally, in order to speed-up the calculations, an adaptive time step method was used by enforcing a maximum control point displacement at each step. 
Electromagnetic calculations. Scattering calculations of a single silicon $\left(\varepsilon_{\mathrm{r}}=12\right)$ scatterer, either on air or over a silicon surface under normal light incidence, have been obtained by FDTD using the freely available Meep package.$^{47}$ Classic perfectly matched layers (PML) where used as boundary conditions, although selected test simulations with adiabatic absorbers where also performed in order to discard any misbehaviour of the PMLs. The scattered field was determined by enclosing the scattering element in a box of flux planes. A planar Gaussian-pulse was used as a source, covering the whole frequency range of interest. A reference simulation without the scatterer was first conducted in order to subtract the incidence fields. The substrate, when considered, was maintained in the reference simulation. In the case of pedestalled structures, during the reference run the spheroid-like section was removed up to narrowest point of the neck, keeping the pedestal and the substrate.

Reflectance calculations of square arrays of pedestalled spheroids over silicon $\left(\varepsilon_{\mathrm{r}}=12\right)$ have been obtained by rigorously coupled wave analysis (RCWA) using the freely available $\mathrm{S}^{4}$ package. ${ }^{48}$ The structure was divided into 50 layers plus silicon as the output medium and 200 orders were considered.

In all optical calculations involving pedestalled structures the geometry resulting from surface diffusion calculations was used as input geometry in both FDTD and RCWA calculations.

\section{Conflicts of interest}

The authors declare no competing financial interest.

\section{Acknowledgements}

The authors acknowledge financial support from the following projects of the Spanish Ministry of Economy and Competitiveness (MINECO): ENE2013-49984-EXP and, co-funded by the European Regional Development Fund (ERDF), ENE2015-74009-JIN. The authors acknowledge fruitful discussions with Prof. F. J. Meseguer and Dr. R. Fenollosa from the Institute of Chemical Technology (ITQ - CSIC).

\section{References}

1. S. Jahani and Z. Jacob, Nature Nanotech. 2015, 11, 23.

2. A. García-Etxarri, R. Gómez-Medina, L. S. Froufe-Pérez, C. López, L. Chantada, F. Scheffold, 
J. Aizpurua, M. Nieto-Vesperinas and J. J. Sáenz, Opt. Express, 2011, 19, 4815.

3. A. I. Kuznetsov, A. E. Miroshnichenko, Y. H. Fu., J. Zhang and B. Luk'yanchuk, Sci. Rep., 2012, 2, 492.

4. A. I. Kuznetsov, A. E. Miroshnichenko, M. L. Brongersma, Y. S. Kivshar and B. Luk’yanchuk, Science, 2016, 354, aag2472.

5. P. Moitra, B. A. Slovick, W. Ii, I. I. Kravchencko, D. P. Briggs, S. Krishnamurthy and J. Valentine, ACS Photonics, 2015, 2, 692.

6. A. S. Schwanecke, V. A. Fedotov, V. V. Khardikov, S. L. Prosvirnin, Y. Chen and N. I. Zheludev, J. Opt. A: Pure Appl. Opt., 2007, 9, L1.

7. J. C. Ginn, I. Brener, D. W. Peters, J. R. Wendt, J. O. Stevens, P. F. Hines, L. I. Basilio, L. K. Warne, J. F. Ihlefeld, P. G. Clem and M. B. Sinclair, Phys Rev. Lett., 2012, 108, 097402.

8. S. Liu, M. B. Sinclair, T. S. Mahony, Y. C. Jun, S. Campione, J. Ginn, D. A. Bender, J. R. Wendt, J. F. Ihlefeld, P. G. Clem, J. B. Wright and I. Brener, Optica, 2014, 4, 250.

9. M. Khorasaninejad, W. T. Chen, R. C. Devlin, J. Oh, A. Y. Zhu and F. Capasso, Science, 2016, 352, 1190.

10. M. I. Shalaev, J. Sun, A. Tsukernik, A. Pandey, K. Nikolskiy and N. M. Litchinitser, Nano Lett., 2015, 15, 6261.

11. M. Decker, I. Staude, M. Falkner, J. Dominguez, D. N. Neshev, I. Brener, T. Pertsch and Y. S. Kivshar, Adv. Opt. Mater., 2015, 3, 813.

12. R. Paniagua-Domínguez, Y. F. Yu, A. E. Miroshnichenko, L. A. Krivitsky, Y. H. Fu, V. Valuckas, L. Gonzaga, Y. T. Toh, A. Y. S Kay, B. Luk’yanchuk and A. I. Kuznetsov, Nat. Comm., 2016, 7, 10362.

13. L. E. Pell, A. D. Schricker, F. V. Mikulec and B. A. Korgel, Langmuir, 2004, 20, 6546.

14. J. T. Harris, J. L. Hueso and B. A. Korgel, Chem. Mater., 2010, 22, 6378.

15. R. Fenollosa, F. Meseguer and M. Tymczenko, Adv. Mater., 2008, 20, 95.

16. R. Fenollosa, F. Ramiro-Manzano, M. Tymczenko and F. Meseguer, J. Mater. Chem., 2010, 20, 
5210.

17. M. Garín, R. Fenollosa and L. Kowalski, Sci. Rep., 2016, 6, 38719.

18. A. B. Evlyukhin, S. M. Novikov, U. Zywietz, R. L. Eriksen, C. Reinhardt, S. I. Bozhevolny and B. N. Chichkov, Nano Lett., 2012, 12, 3749.

19. U. Zywietz, A. B. Evlyukhin, C. Reinhardt and B. N. Chichkov, Nat. Commun., 2014, 5, 3402.

20. A. Gumennik, L. Wei, G. Lestoquoy, A. M. Stolyarov, X. Jia, P. H. Rekemeyer, M. J. Smith, X. Liang, B. J.-B Grena, S. G. Johnson, S. Gradečak, A. F. Abouraddy, J. D. Joannopoulos and Y. Fink, Nat. Commun., 2013, 4, 2216.

21. Y. H. Fu, A. I. Kuznetsov, A. E. Miroshnichenko, Y. F. Yu and B. Luk'yanchuk, Nat. Commun., 2013, 4, 1527.

22. M. I. Tribelsky, J.-M. Geffrin, A. Litman, C. Eyraud and F. Moreno, Sci. Rep., 2015, 5, 12288.

23. P. Albella, T. Shibanuma and S. A. Maier, Sci. Rep., 2015, 5, 18322.

24. Y. O. Yilmaz, A. Demir and A. Serpengüzel, IEEE Photonics Technol. Lett., 2005, 17, 1662.

25. A. Serpengüzel and A. Demir, Semicond. Sci. Technol., 2008, 23, 064009.

26. E. Xifré-Perez, J. D. Domenech, R. Fenollosa, P. Muñoz, J. Capmany and F. Meseguer, Opt. Exp., 2011, 19, 3185.

27. M. Garín, R. Fenollosa, R. Alcubilla, L. Shi, L. F. Marsal and F. Meseguer, Nat. Commun., 2014, 5, 3440.

28. M. Garín, R. Fenollosa, P. Ortega and F. Meseguer, J. Appl. Phys., 2016, 119, 033101.

29. O. Gil-Castell, J.D. Badia, R. Teruel-Juanes, I. Rodriguez, F. Meseguer and A. Ribes-Greus, Eur. Polym. J., 2015, 70, 247.

30. P. Albella, M. A. Poyli, M. K. Schmidt, S. A. Maier, F. Moreno, J. J. Sáenz, and J. Aizpurua, J. Phys. Chem. C, 2013, 117, 13573.

31. P. Albella, R. Alcaraz de la Osa, F. Moreno, and S. A. Maier, ACS Photonics, 2014, 1, 524.

32. I. Rodriguez, L. Shi, X. Lu, B. A. Korgel, R. A. Alvarez-Puebla and F. Meseguer, Nanoscale, 
2014, 6, 5666.

33. M. Caldarola, P. Albella, E. Cortés, M. Rahmani, T. Roschuk, g. Grinblat, R. F. Oulton, A. V. Bragas, and S. A. Maier, Nature Commun., 2015, 6, 7915.

34. A. Butakov and J. A. Schuller, Sci. Rep., 2016, 6, 38487.

35. P. A. Dmitriev, S. V. Makarov, V. A. Milichko, I. S. Mukhin, A. S. Gudovskikh, A. A. Sitnikova, A. K. Samusev, A. E. Krasnok and P. A. Belov, Nanoscale, 2016, 8, 5043.

36. W. W. Mullins, J. Appl. Phys., 1957, 28, 333.

37. F. A. Nichols and W. W. Mullins, J. Appl. Phys., 1695, 36, 1826.

38. M. Garín, C. Jin, D. Cardador, , T. Trifonov and R. Alcubilla, Sci. Rep., 2017, 7, 7233.

39. B. Slovick, Z. Gang Yu, M. Berding and S. Krishnamurthy, Phys. Rev. B: Condens. Matter Mater. Phys., 2013, 88, 165116.

40. I. Staude, A. E. Miroshnichenko, M. Decker, N. T. Fofang, S. Liu, E. Gonzalez, J. Dominguez, T. S. Luk, D. N. Neshev, I. Brener, and Y. Kivshar, ACS Nano, 2013, 9, 7824.

41. Y. F. Yu, A. Y. Zhu, R. Paniagua-Domínguez, Y. H. Fu, B. Luk’yanchuk, and A. I. Kuznetsov, Laser Photonics Rev., 2015, 9, 412.

42. T. Shibanuma, S. A. Maier, and P. Albella, Appl. Phys. Lett., 2018, 112, 063103.

43. L. Shi, R. Fenollosa, T. U. Tuzer and F. Meseguer, ACS Photonics, 2014, 1, 408.

44. P. Spinelli, M.A. Verschuuren and A. Polman, Nat. Commun., 2012, 3, 692.

45. Z. Dong, J. Ho, Y. F. Yu, Y. H. Fu, R. Paniagua-Dominguez, S. Wang, A. I. Kuznetsov and J. K. W. Yang, Nano Lett., 2017, 17, 7620.

46. M. F. Castez, P. C. dos Santos Claro, P. L. Schilardi, G. Andreasen, and R. C. Salvarezza, J. Phys. Chem. C, 2010, 114, 4603.

47. A.F. Oskooi, D. Roundy, M. Ibanescu, P. Bermel, J. D. Joannopoulos and S. G. Johnson, Comput. Phys. Commun., 2010, 181, 687.

48. V. Liu and S. Fan, Comput. Phys. Comm., 2012, 183, 2233. 